\title{
Non-Archimedean Radial Calculus: Volterra Operator and Laplace Transform
}

\author{
Anatoly N. Kochubei \\ Institute of Mathematics, \\ National Academy of Sciences of Ukraine, \\ Tereshchenkivska 3, Kiev, 01024 Ukraine \\ E-mail: kochubei@imath.kiev.ua
}

\begin{abstract}
In an earlier paper (A. N. Kochubei, Pacif. J. Math. 269 (2014), 355-369), the author considered a restriction of Vladimirov's fractional differentiation operator $D^{\alpha}, \alpha>0$, to radial functions on a non-Archimedean field. In particular, it was found to possess such a right inverse $I^{\alpha}$ that the appropriate change of variables reduces equations with $D^{\alpha}$ (for radial functions) to integral equations whose properties resemble those of classical Volterra equations. In other words, we found, in the framework of non-Archimedean pseudo-differential operators, a counterpart of ordinary differential equations. In the present paper, we begin an operator-theoretic investigation of the operator $I^{\alpha}$, and study a related analog of the Laplace transform.
\end{abstract}

Key words: fractional differentiation operator; non-Archimedean local field; radial functions; Volterra operator; Laplace transform

MSC 2020. Primary: 47G10. Secondary: 11S80, 35S10, 43A32. 


\section{Introduction}

The basic linear operator defined on real- or complex-valued functions on a non-Archimedean local field $K$ (such as $K=\mathbb{Q}_{p}$, the field of $p$-adic numbers) is the Vladimirov pseudo-differential operator $D^{\alpha}, \alpha>0$, of fractional differentiation [19]; for further development of this subject see [1, 8, 11, 12, 22, 3]. Note also the recent publications devoted to applications in geophysical models and to the study of related nonlinear equations [17, 9, 18, 10].

It was found in [13] that properties of $D^{\alpha}$ become much simpler on radial functions. Moreover, in this case it was found to possess a right inverse $I^{\alpha}$, which can be seen as a $p$-adic counterpart of the Riemann-Liouville fractional integral or, for $\alpha=1$, the classical anti-derivative. The change of an unknown function $u=I^{\alpha} v$ reduces the Cauchy problem for an equation with the radial restriction of $D^{\alpha}$ to an integral equation with properties resembling those of classical Volterra equations. In other words, we found, in the framework of non-Archimedean pseudodifferential operators, a counterpart of ordinary differential equations. In [13, we studied linear equations of this kind; nonlinear ones were investigated in [15]. Note that radial functions appear as exact solutions of the $p$-adic analog of the classical porous medium equation [9].

In this paper we study the operator $I^{1}$ on the ring of integers $O \subset K$ as an object of operator theory. The operator $I^{1}$ on $L^{2}(O)$ happens to be a sum of a bounded selfadjoint operator and a simple Volterra operator $I_{0}^{1}$ with a rank two imaginary part $J$, such that $\operatorname{tr} J=0$. The characteristic matrix-function $W(z)$ of $I_{0}^{1}$ is such that $W\left(z^{-1}\right)$ is, in contrast to classical examples, an entire matrix function of zero order.

While the theory of Volterra operators and their characteristic functions is well-developed (see [5, 4, 6, 16, 21]), properties of the above operator are very different from those known for operators of classical analysis and their generalizations. Therefore, while $I^{1}$ and $I_{0}^{1}$ are just specific examples, they create a framework for future studies in this area.

Another subject touched in this paper is a version of the Laplace transform. The classical Laplace transform is based on the function $x \mapsto e^{-\lambda x}$ satisfying an obvious differential equation. A similar equation involving $D^{\alpha}$ has a unique radial solution [12]. This leads to a definition of the Laplace type transform in the above framework. We prove a uniqueness theorem and the inversion formula for this transform.

\section{Preliminaries}

2.1. Local fields. Let $K$ be a non-Archimedean local field, that is a non-discrete totally disconnected locally compact topological field. It is well known that $K$ is isomorphic either to a finite extension of the field $\mathbb{Q}_{p}$ of $p$-adic numbers (if $K$ has characteristic 0 ), or to the field of formal Laurent series with coefficients from a finite field, if $K$ has a positive characteristic. For a summary of main notions and results regarding local fields see, for example, [11.

Any local field $K$ is endowed with an absolute value $|\cdot|_{K}$, such that $|x|_{K}=0$ if and only if $x=0,|x y|_{K}=|x|_{K} \cdot|y|_{K},|x+y|_{K} \leq \max \left(|x|_{K},|y|_{K}\right)$. Denote $O=\left\{x \in K:|x|_{K} \leq 1\right\}$, $P=\left\{x \in K:|x|_{K}<1\right\}$. $O$ is a subring of $K$, and $P$ is an ideal in $O$ containing such an element $\beta$ that $P=\beta O$. The quotient $\operatorname{ring} O / P$ is actually a finite field; denote by $q$ its cardinality. We will always assume that the absolute value is normalized, that is $|\beta|_{K}=q^{-1}$. The normalized absolute value takes the values $q^{N}, N \in \mathbb{Z}$. Note that for $K=\mathbb{Q}_{p}$ we have 
$\beta=p$ and $q=p$; the $p$-adic absolute value is normalized.

The additive group of any local field is self-dual, that is if $\chi$ is a fixed non-constant complexvalued additive character of $K$, then any other additive character can be written as $\chi_{a}(x)=$ $\chi(a x), x \in K$, for some $a \in K$. Below we assume that $\chi$ is a rank zero character, that is $\chi(x) \equiv 1$ for $x \in O$, while there exists such an element $x_{0} \in K$ that $\left|x_{0}\right|_{K}=q$ and $\chi\left(x_{0}\right) \neq 1$.

The above duality is used in the definition of the Fourier transform over $K$. Denoting by $d x$ the Haar measure on the additive group of $K$ (normalized in such a way that the measure of $O$ equals 1 ) we write

$$
\widetilde{f}(\xi)=\int_{K} \chi(x \xi) f(x) d x, \quad \xi \in K,
$$

where $f$ is a complex-valued function from $L_{1}(K)$. As usual, the Fourier transform $\mathcal{F}$ can be extended from $L_{1}(K) \cap L_{2}(K)$ to a unitary operator on $L_{2}(K)$. If $\mathcal{F} f=\widetilde{f} \in L_{1}(K)$, we have the inversion formula

$$
f(x)=\int_{K} \chi(-x \xi) \tilde{f}(\xi) d \xi .
$$

Working with functions on $K$ and operators upon them we often use standard integration formulas; see [11, 19]. The simplest of them are as follows:

$$
\begin{gathered}
\int_{|x|_{K} \leq q^{n}} d x=q^{n} ; \quad \int_{|x|_{K}=q^{n}} d x=\left(1-\frac{1}{q}\right) q^{n} . \\
\int_{|x|_{K} \leq q^{n}}|x|_{K}^{\alpha-1} d x=\frac{1-q^{-1}}{1-q^{-\alpha}} q^{\alpha n} ; \quad \text { here and above } n \in \mathbb{Z}, \alpha>0 .
\end{gathered}
$$

A function $f: K \rightarrow \mathbb{C}$ is said to be locally constant, if there exists such an integer $l$ that for any $x \in K$

$$
f\left(x+x^{\prime}\right)=f(x), \quad \text { whenever }\left|x^{\prime}\right| \leq q^{-l} .
$$

The vector space $\mathcal{D}(K)$ of all locally constant functions with compact supports is used as a space of test functions in analysis on $K$. Note that the Fourier transform preserves $\mathcal{D}(K)$. There exists a well-developed theory of distributions on local fields; see [1, 11, 19].

2.2. Vladimirov's operator. On a test function $\varphi \in \mathcal{D}(K)$, the fractional differentiation operator $D^{\alpha}, \alpha>0$, is defined as

$$
\left(D^{\alpha} \varphi\right)(x)=\mathcal{F}^{-1}\left[|\xi|_{K}^{\alpha}(\mathcal{F}(\varphi))(\xi)\right](x) .
$$

Note that $D^{\alpha}$ does not preserve $\mathcal{D}(K)$; see [1] regarding the spaces of test functions and distributions preserved by this operator.

The operator $D^{\alpha}$ can also be represented as a hypersingular integral operator:

$$
\left(D^{\alpha} \varphi\right)(x)=\frac{1-q^{\alpha}}{1-q^{-\alpha-1}} \int_{K}|y|_{K}^{-\alpha-1}[\varphi(x-y)-\varphi(x)] d y
$$


[11, 19]. In contrast to (2.1), the expression in the right of (2.2) makes sense for wider classes of functions. In particular, $D^{\alpha}$ is defined on constant functions and annihilates them. Denote for brevity $\theta_{\alpha}=\frac{1-q^{\alpha}}{1-q^{-\alpha-1}}$.

Below we consider the operator $D^{\alpha}$ on a radial function $u=u\left(|x|_{K}\right)$; here we identify the function $x \mapsto u\left(|x|_{K}\right)$ on $K$ with the function $|x|_{K} \mapsto u\left(|x|_{K}\right)$ on $q^{\mathbb{Z}}$. This abuse of notation does not lead to confusion.

The explicit expression of $D^{\alpha} u$ for a radial function $u$ satisfying some growth restrictions near the origin and infinity was found in [13]. If $u=u\left(|x|_{K}\right)$ is such that

$$
\sum_{k=-\infty}^{m} q^{k}\left|u\left(q^{k}\right)\right|<\infty, \quad \sum_{l=m}^{\infty} q^{-\alpha l}\left|u\left(q^{l}\right)\right|<\infty
$$

for some $m \in \mathbb{Z}$, then for each $n \in \mathbb{Z}$ the expression in the right-hand side of (2.2) with $\varphi(x)=u\left(|x|_{K}\right)$ exists for $|x|_{K}=q^{n}$, depends only on $|x|_{K}$, and

$$
\begin{aligned}
\left(D^{\alpha} u\right)\left(q^{n}\right)=\theta_{\alpha}\left(1-\frac{1}{q}\right) q^{-(\alpha+1) n} \sum_{k=-\infty}^{n-1} q^{k} u\left(q^{k}\right)+q^{-\alpha n-1} \frac{q^{\alpha}+q-2}{1-q^{-\alpha-1}} u\left(q^{n}\right) & \\
& +\theta_{\alpha}\left(1-\frac{1}{q}\right) \sum_{l=n+1}^{\infty} q^{-\alpha l} u\left(q^{l}\right) .
\end{aligned}
$$

Under the conditions (2.3), the expression (2.4) agrees also with the definition of $D^{\alpha}$ in terms of Bruhat-Schwartz distributions (see Chapter 2 of [19]).

2.3. The regularized integral. The fractional integral mentioned in Introduction, was defined in [13] initially for $\varphi \in \mathcal{D}(K)$ as follows:

$$
\left(I^{\alpha} \varphi\right)(x)=\left(D^{-\alpha} \varphi\right)(x)-\left(D^{-\alpha} \varphi\right)(0)
$$

where $D^{-\alpha}$ is the right inverse of $D^{\alpha}$ introduced by Vladimirov [19]:

$$
\begin{gathered}
\left(D^{-\alpha} \varphi\right)(x)=\left(f_{\alpha} * \varphi\right)(x)=\frac{1-q^{-\alpha}}{1-q^{\alpha-1}} \int_{K}|x-y|_{K}^{\alpha-1} \varphi(y) d y, \quad \alpha \neq 1 \\
\left(D^{-1} \varphi\right)(x)=\frac{1-q}{q \log q} \int_{K} \log |x-y|_{K} \varphi(y) d y .
\end{gathered}
$$

$D^{-1}$ is a right inverse to $D^{1}$ only on such functions $\varphi$ that

$$
\int_{K} \varphi(x) d x=0 .
$$

On such a function $\varphi$ we have also $D^{-1} D^{1} \varphi=\varphi$.

The above definition $(*)$ leads to explicit expressions

$$
\left(I^{\alpha} \varphi\right)(x)=\frac{1-q^{-\alpha}}{1-q^{\alpha-1}} \int_{|y|_{K} \leq|x|_{K}}\left(|x-y|_{K}^{\alpha-1}-|y|_{K}^{\alpha-1}\right) \varphi(y) d y, \quad \alpha \neq 1
$$


and

$$
\left(I^{1} \varphi\right)(x)=\frac{1-q}{q \log q} \int_{|y|_{K} \leq|x|_{K}}\left(\log |x-y|_{K}-\log |y|_{K}\right) \varphi(y) d y .
$$

Note that the integrals are taken, for each fixed $x \in K$, over bounded sets, and $\left(I^{\alpha} \varphi\right)(0)=0$. These properties are different from those of the anti-derivatives $D^{-\alpha}$ studied in [19].

Let $u=u\left(|x|_{K}\right)$ be a radial function, such that

$$
\sum_{k=-\infty}^{m} \max \left(q^{k}, q^{\alpha k}\right)\left|u\left(q^{k}\right)\right|<\infty, \quad \text { if } \alpha \neq 1
$$

and

$$
\sum_{k=-\infty}^{m}|k| q^{k}\left|u\left(q^{k}\right)\right|<\infty, \quad \text { if } \alpha=1
$$

for some $m \in \mathbb{Z}$. Then [13] $I^{\alpha} u$ exists, it is a radial function, and for any $x \neq 0$,

$$
\left(I^{\alpha} u\right)\left(|x|_{K}\right)=q^{-\alpha}|x|_{K}^{\alpha} u\left(|x|_{K}\right)+\frac{1-q^{-\alpha}}{1-q^{\alpha-1}} \int_{|y|_{K}<|x|_{K}}\left(|x|_{K}^{\alpha-1}-|y|_{K}^{\alpha-1}\right) u\left(|y|_{K}\right) d y, \quad \alpha \neq 1
$$

and

$$
\left(I^{1} u\right)\left(|x|_{K}\right)=q^{-1}|x|_{K} u\left(|x|_{K}\right)+\frac{1-q}{q \log q} \int_{|y|_{K}<|x|_{K}}\left(\log |x|_{K}-\log |y|_{K}\right) u\left(|y|_{K}\right) d y .
$$

On an appropriate class of radial functions, $I^{\alpha}$ is a right inverse to $D^{\alpha}$ [13. An important difference between $D^{-\alpha}$ and $I^{\alpha}$ is the bounded integration domain in the integral formulas for $I^{\alpha}$.

\subsection{Radial eigenfunctions of $D^{\alpha}$.}

The operator $D^{\alpha}$ defined initially on $\mathcal{D}(K)$ is, after its closure in $L^{2}(K)$, a selfadjoint operator with a pure point spectrum $\left\{q^{\alpha N}, N \in \mathbb{Z}\right\}$ of infinite multiplicity and a single limit point zero.

It was shown in [12] that for each $N \in \mathbb{Z}$, there exists a unique (up to the multiplication by a constant) radial eigenfunction

$$
v_{N}\left(|x|_{K}\right)= \begin{cases}1, & \text { if }|x|_{K} \leq q^{-N} \\ -\frac{1}{q-1}, & \text { if }|x|_{K}=q^{-N+1} \\ 0, & \text { if }|x|_{K} \geq q^{-N+2}\end{cases}
$$

corresponding to the eigenvalue $\lambda=q^{\alpha N}$. Below we interpret this function as an analog of the classical exponential function $x \mapsto e^{-\lambda x}$. Note that $v_{N} \in \mathcal{D}(K)$; this is a purely nonArchimedean phenomenon reflecting the unusual topological property of $K$, its total disconnectedness.

The operator $D_{O}^{\alpha}$ in the space $L^{2}(O)$ on the ring of integers (unit ball) $O$ is defined as follows. Extend a function $\varphi \in \mathcal{D}(O)$ (that is a function $\varphi \in \mathcal{D}(K)$ supported in $O$ ) onto $K$ by 
zero. Apply $D^{\alpha}$ and consider the resulting function on $O$. After the closure in $L^{2}(O)$ we obtain a selfadjoint operator $D_{O}^{\alpha}$ with a discrete spectrum [11, 19] (here we do not touch different definitions from [2] and [14]).

Denote by $\mathcal{H}$ the subspace in $L^{2}(O)$ consisting of radial functions. The functions $v_{N}$, $N=1,2, \ldots$ belong to $\mathcal{H}$, as well as the function

$$
v_{0}\left(|x|_{K}\right) \equiv 1, \quad|x|_{K} \leq 1
$$

By the definition of $D_{O}^{\alpha}$, the functions $v_{N}$ are its eigenfunctions corresponding to the eigenvalues $q^{\alpha N}$. As for $v_{0}$, it is also an eigenfunction, with the eigenvalue $\mu_{0}=\frac{q-1}{q^{\alpha+1}-1} q^{\alpha}$ [11, 19]. Therefore $\left\{v_{N}\right\}_{N \geq 0}$ is an orthonormal system in $L^{2}(O)$, hence in $\mathcal{H}$.

We have $\left\|v_{0}\right\|=1(\|\cdot\|$ is the norm in $\mathcal{H})$,

$$
\begin{gathered}
\left\|v_{N}\right\|^{2}=\int_{|x|_{K} \leq q^{-N}} d x+(q-1)^{-2} \int_{|x|_{K}=q^{-N+1}} d x=q^{-N}+(q-1)^{-2} q^{-N+1}\left(1-\frac{1}{q}\right)=(q-1)^{-1} q^{-N}, \\
\quad \int_{|x|_{K} \leq 1} v_{N}\left(|x|_{K}\right) d x=0, \quad N \geq 1 .
\end{gathered}
$$

Therefore the functions

$$
e_{0}\left(|x|_{K}\right) \equiv 1 ; \quad e_{N}\left(|x|_{K}\right)=(q-1)^{1 / 2} q^{N / 2} v_{N}\left(|x|_{K}\right), \quad N \geq 1
$$

form an orthonormal system in $\mathcal{H}$.

Lemma 1. The system $\left\{e_{N}\right\}_{N \geq 0}$ is an orthonormal basis in $\mathcal{H}$.

Proof. Let $u \in \mathcal{H}$ be orthogonal to all the functions $e_{N}$. Then

$$
\int_{|x|_{K} \leq 1} u\left(|x|_{K}\right) d x=0
$$

so that

$$
\sum_{j=-\infty}^{0} u\left(q^{j}\right) q^{j}=0
$$

and

$$
\int_{|x|_{K} \leq q^{-N}} u\left(|x|_{K}\right) d x-(q-1)^{-1} \int_{|x|_{K}=q^{-N+1}} u\left(|x|_{K}\right) d x=0
$$

so that

$$
\sum_{j=-\infty}^{-N} u\left(q^{j}\right) q^{j}-(q-1)^{-1} u\left(q^{-N+1}\right)=0, \quad N=1,2, \ldots
$$


Subtracting from (2.8) the equality (2.9) with $N=1$, we find that $u(1)=0$. Now the equality (2.9) with $N=1$ takes the form

$$
\sum_{j=-\infty}^{-1} u\left(q^{j}\right) q^{j}=0
$$

while (2.9) with $N=2$ yields

$$
\sum_{j=-\infty}^{-2} u\left(q^{j}\right) q^{j}-(q-1)^{-1} u\left(q^{-1}\right)=0
$$

Subtracting we obtain that $u\left(q^{-1}\right)=0$.

Repeating the above reasoning we find that $u=0$.

Another (obvious) orthonormal basis in $\mathcal{H}$ is

$$
f_{n}\left(|x|_{K}\right)=\left\{\begin{array}{ll}
\left(1-\frac{1}{q}\right)^{-1 / 2} q^{n / 2}, & \text { if }|x|_{K}=q^{-n} ; \\
0, & \text { elsewhere, }
\end{array} \quad n=0,1,2, \ldots\right.
$$

The next result is of some independent interest.

Proposition 1. The set of "polynomials"

$$
u\left(|x|_{K}\right)=\sum_{n=1}^{N} a_{n}|x|_{K}^{n}, \quad a_{n} \in \mathbb{C}, \quad N \geq 1,
$$

is dense in $\mathcal{H}$.

Proof. Suppose that a function $F \in \mathcal{H}$ is orthogonal to all the functions $X_{l}\left(|x|_{K}\right)=|x|_{K}^{l}$, $l \geq 1$. Using the basis (2.10), write

$$
F=\sum_{n=0}^{\infty} c_{n} f_{n}, \quad\left\{c_{n}\right\} \in l^{2}
$$

We have

$$
\left\langle X_{l}, f_{n}\right\rangle=\left(1-\frac{1}{q}\right)^{-1 / 2} q^{n / 2} \int_{|x|_{K}=q^{-n}}|x|_{K}^{l} d x=\left(1-\frac{1}{q}\right)^{1 / 2} q^{-n / 2-n l}
$$

so that

$$
\left\langle F, X_{l}\right\rangle=\left(1-\frac{1}{q}\right)^{1 / 2} \sum_{n=0}^{\infty} c_{n} q^{-n / 2-n l}=0, \quad l=1,2, \ldots
$$

Denoting $\beta=q^{-1}, b_{n}=c_{n} q^{-n / 2}$, we see that the vector $\left(b_{0}, b_{1}, b_{2}, \ldots\right) \in l^{2}$ is orthogonal in $l^{2}$ to each vector $\left(1, \beta^{l}, \beta^{2 l}, \ldots\right), l \geq 1$. It is known ([7], Problem 6) that the set of all these vectors is total in $l^{2}$, so that $F=0$.

In fact, the above reasoning proves the density of polynomials (2.11) in a wider weighted space determined by the condition $\left\{c_{n} q^{-n / 2}\right\} \in l^{2}$. 


\section{$3 \quad$ Integration Operators}

3.1. The operator $I^{1}$. Let us study $I^{1}$ as an operator in $\mathcal{H}$, find its matrix representation with respect to the basis $\left\{e_{N}\right\}$ and investigate the spectrum of $I^{1}$.

Proposition 2. The operator $I^{1}$ has the matrix representation

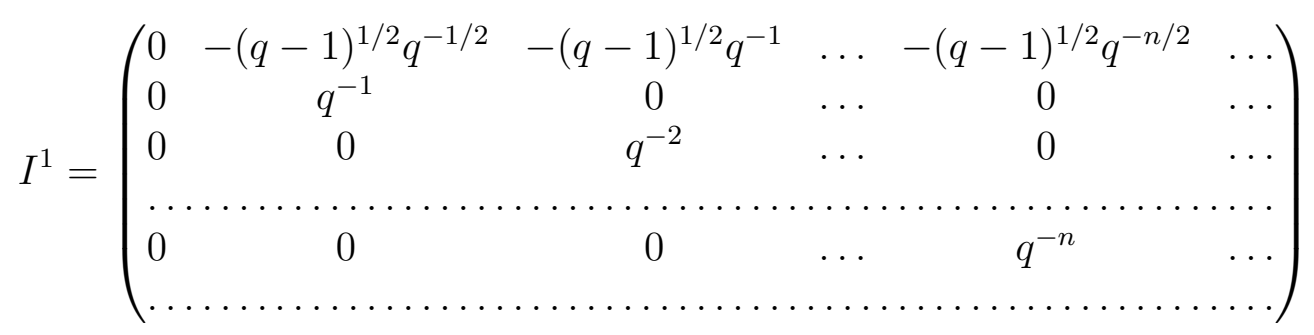

(only the first row and the principal diagonal have nonzero elements). $I^{1}$ is a Hilbert-Schmidt operator. Apart from being a point of essential spectrum, $\lambda=0$ is a simple eigenvalue. In addition, $I^{1}$ has simple eigenvalues $\lambda_{m}=q^{-m}, m=1,2, \ldots$

Proof. Since the integral of each function $e_{N}, N \geq 1$, equals zero, we have $D^{-1} D^{1} e_{N}=e_{N}$. On the other hand, $D^{1} e_{N}=q^{N} e_{N}$, so that $D^{-1} e_{N}=q^{-N} e_{N}$, and by the definition (*) of $I^{1}$,

$$
I^{1} e_{N}=q^{-N} e_{N}-(q-1)^{1 / 2} q^{-N / 2} e_{0}, \quad N=1,2, \ldots
$$

Next, $\left(I^{1} e_{0}\right)\left(|x|_{K}\right),|x|_{K} \leq 1$, depends only on the values of $e_{0}$ for $|x|_{K} \leq 1$. Let $f(x) \equiv 1$, $x \in K$. Then $I^{1} f=0$ [13], so that

$$
I^{1} e_{0}=0 \text { in } \mathcal{H}
$$

The equalities (3.1) and (3.2) imply the required matrix representation, which implies the Hilbert-Schmidt property.

Let us find the eigenvalues of $I^{1}$. As we have seen, $I^{1} e_{0}=0$. Suppose that

$$
u=\sum_{n=0}^{\infty} c_{n} e_{n}, \quad\left\{c_{n}\right\} \in l^{2}, \quad I^{1} u=\lambda u
$$

By (3.1) and (3.2),

$$
I^{1} u=\sum_{n=1}^{\infty} q^{-n} c_{n} e_{n}-\left[\sum_{n=1}^{\infty}(q-1)^{1 / 2} q^{-n / 2} c_{n}\right] e_{0}
$$

and we find that

$$
\left\{\begin{array}{l}
\lambda c_{0}=-(q-1)^{1 / 2} \sum_{n=1}^{\infty} q^{-n / 2} c_{n} \\
\lambda c_{n}=q^{-n} c_{n}, \quad n \geq 1 .
\end{array}\right.
$$

A nonzero value of $c_{n}(n \geq 1)$ is possible only for a single index $n=m$, and in this case $\lambda=q^{-m}$. Then the first equation in (3.3) gives $c_{m}=-(q-1)^{-1 / 2} q^{-m / 2} c_{0}$, so that

$$
u=c_{0} e_{0}-(q-1)^{-1 / 2} q^{-m / 2} c_{0} e_{m}
$$


is the unique (up to the multiplication by a constant) eigenfunction.

3.2. A local representation. The definition $(*)$ of the operator $I^{\alpha}$ involves operators in $L^{2}(K)$; then we make restrictions to $L^{2}(O)$ and $\mathcal{H}$. In this section we show, for the case where $\alpha=1$, that a similar representation containing only operators in $L^{2}(O)$ is also possible.

Theorem 1. If $u \in L^{2}(O)$, then

$$
\left(I^{1} u\right)(x)=\left(\left(D_{O}^{1}\right)^{-1} u\right)(x)-\left(\left(D_{O}^{1}\right)^{-1} u\right)(0) .
$$

Proof. In [14], we found the resolvent $\left(D_{O}^{1}-\mu_{0}+\mu\right)^{-1}$ where $\mu_{0}=\frac{q}{q+1}$ (the first eigenvalue of $\left.D_{O}^{1}\right), \mu>0$. In [14, in connection with nonlinear equations, we considered operators in $L^{1}(O)$, but the result is valid for $L^{2}(O)$ too. For $\mu=\mu_{0}$,

$$
\left(D_{O}^{1}\right)^{-1} u(x)=\int_{|\xi|_{K} \leq 1} \mathcal{K}(x-\xi) u(\xi) d \xi+\mu_{0}^{-1} \int_{|\xi|_{K} \leq 1} u(\xi) d \xi
$$

where for $|x|_{K}=q^{m}, m \leq 0$,

$$
\mathcal{K}(x)=\int_{q \leq|\eta|_{K} \leq q^{-m+1}}|\eta|_{K}^{-1} \chi(\eta x) d \eta .
$$

Using the well-known integration formula (see, for example, Section 1.5 in [11]), we get

$$
\begin{array}{rl}
\mathcal{K}(x)=\sum_{j=1}^{-m+1} q^{-j} \int_{|\eta|_{K}=q^{j}} \chi(\eta x) d \eta=\left(1-\frac{1}{q}\right) \sum_{j=1}^{-m} & 1-q^{-1} \\
& =-\left(1-\frac{1}{q}\right) m-q^{-1}=\frac{1-q}{q \log q} \log |x|_{K}-q^{-1} .
\end{array}
$$

By (3.5),

$$
\left(D_{O}^{1}\right)^{-1} u(x)=\frac{1-q}{q \log q} \int_{|\xi|_{K} \leq 1} \log |x-\xi|_{K} u(\xi) d \xi+\int_{|\xi|_{K} \leq 1} u(\xi) d \xi .
$$

Comparing with the expression for $I^{1}$ and noticing that $|x-\xi|_{K}-|\xi|_{K}=0$, if $|\xi|_{K}>|x|_{K}$, we obtain (3.4).

3.3. The Volterra operator. Let us consider the integral part of (2.5) , the operator

$$
\left(I_{0}^{1} u\right)(x)=\frac{1-q}{q \log q} \int_{|y|_{K}<|x|_{K}}\left(\log |x|_{K}-\log |y|_{K}\right) u\left(|y|_{K}\right) d y .
$$

Recall [5] that a compact operator is called a Volterra operator, if its spectrum consists of the unique point $\lambda=0$. An operator $A$ is called simple, if $A$ and $A^{*}$ have no common nontrivial 
invariant subspace, on which these operators coincide. It is known [5] that a Volterra operator $A$ is simple, if and only if the equations $A f=0$ and $A^{*} f=0$ have no common nontrivial solutions.

The main technical tool in the study of $I_{0}^{1}$ is the identity [13]

$$
\int_{|y|_{K}<|x|_{K}}\left(\log |x|_{K}-\log |y|_{K}\right)|y|_{K}^{m} d y=d_{m}|x|_{K}^{m+1}, \quad m=0,1,2, \ldots,
$$

where $0<d_{m} \leq A q^{-m}, A>0$ does not depend on $m$.

Theorem 2. The operator $I_{0}^{1}$ in $\mathcal{H}$ is a simple Volterra operator with a rank 2 imaginary part $J=\frac{1}{2 i}\left(A-A^{*}\right)$, such that $\operatorname{tr} J=0$.

Proof. 1) Suppose that $I_{0}^{1} u=\lambda u, u \in \mathcal{H}, \lambda \in \mathbb{C}, \lambda \neq 0$. Then for $|x|_{K} \leq 1$,

$$
\begin{aligned}
\left|u\left(|x|_{K}\right)\right| \leq \frac{c}{|\lambda|}\|u\|_{L^{2}(O)}\left[\int_{\left\lfloor\left. y\right|_{K}<|x|_{K}\right.}\left(\log |x|_{K}-\log |y|_{K}\right)^{2} d y\right]^{1 / 2} & \leq \frac{c}{|\lambda|}\|u\|_{L^{2}(O)}\left[q^{-1}|x|_{K}\left(\log |x|_{K}\right)^{2}\right]^{1 / 2} \leq H
\end{aligned}
$$

where $c=\frac{q-1}{q \log q}, H$ is a positive constant.

This implies the estimate

$$
\left|u\left(|x|_{K}\right)\right| \leq \frac{c H}{|\lambda|} \int_{|y|_{K}<|x|_{K}}\left(\log |x|_{K}-\log |y|_{K}\right) d y
$$

and by the identity (3.6) with $m=0$,

$$
\left|u\left(|x|_{K}\right)\right| \leq \frac{c H A}{|\lambda|}|x|_{K} .
$$

Similarly, the identity (3.6) with $m=1$ gives

$$
\left|u\left(|x|_{K}\right)\right| \leq \frac{c^{2} H A^{2}}{|\lambda|^{2}} q^{-1}|x|_{K}^{2},
$$

and we find by induction that

$$
\left|u\left(|x|_{K}\right)\right| \leq \frac{c^{m+1} H A^{m+1}}{|\lambda|^{m+1}} q^{-1} q^{-2} \cdots q^{-m+1}|x|_{K}^{m+1}
$$

for an arbitrary natural number $m$.

Note that

$$
q^{-1} q^{-2} \cdots q^{-m+1}=\left(\frac{1}{q}\right)^{m(m-1) / 2}
$$


Together with (3.7), this shows that $u \equiv 0$.

2) It follows from the definition of $I_{0}^{1}$ that $\lambda=0$ is an eigenvalue corresponding to the eigenfunction

$$
u_{0}\left(|x|_{K}\right)= \begin{cases}1, & \text { if }|x|_{K}=1 \\ 0, & \text { if }|x|_{K}<1 .\end{cases}
$$

Let us show that $\lambda=0$ does not correspond to other eigenfunctions.

Suppose that $I_{0}^{1} \varphi=0$ for some $\varphi \in \mathcal{H}$, so that

$$
\sum_{j=-\infty}^{n-1}(n-j) q^{j} \varphi\left(q^{j}\right)=0, \quad n=0,-1,-2, \ldots
$$

Together with (3.9), consider a similar equality with $n-1$ substituted for $n$, that is

$$
\sum_{j=-\infty}^{n-2}(n-1-j) q^{j} \varphi\left(q^{j}\right)=0, \quad n=0,-1,-2, \ldots
$$

Subtracting (3.9) from (3.10) we find that

$$
\sum_{j=-\infty}^{n-1} q^{j} \varphi\left(q^{j}\right)=0, \quad n=0,-1,-2, \ldots,
$$

that is, in particular,

$$
\begin{aligned}
& q^{n-1} \varphi\left(q^{n-1}\right)+q^{n-2} \varphi\left(q^{n-2}\right)+\cdots=0, \\
& q^{n-2} \varphi\left(q^{n-2}\right)+q^{n-3} \varphi\left(q^{n-3}\right)+\cdots=0,
\end{aligned}
$$

Subtracting the second equality from the first one, we find that $\varphi\left(q^{-1}\right)=\varphi\left(q^{-2}\right)=\ldots=0$, so that $\varphi$ is proportional to the eigenfunction (3.8).

3) The imaginary part $J$ has the following matrix representation with respect to the basis $\left\{e_{N}\right\}$ :

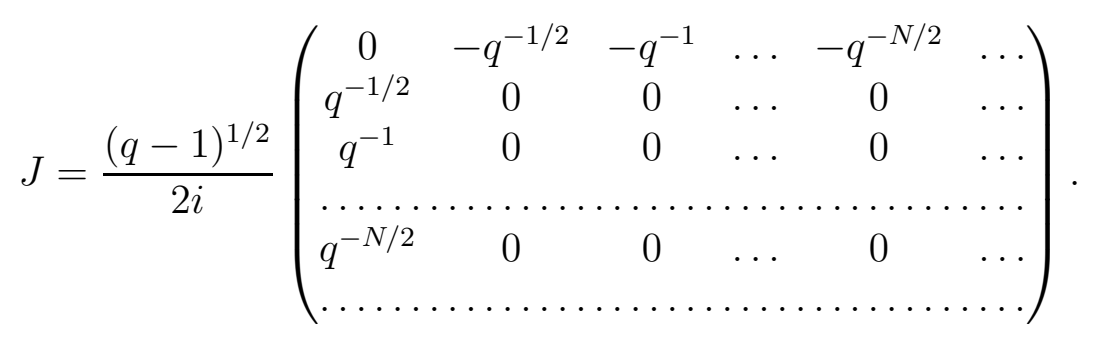

It is easy to write an integral representation

$$
(J u)\left(|x|_{K}\right)=\frac{1-q}{2 i q \log q} \int_{|y|_{k} \leq 1}\left(\log |x|_{K}-\log |y|_{K}\right) u\left(|y|_{K} d y,\right.
$$

that is

$$
(J u)\left(|x|_{K}\right)=\frac{1-q}{2 i q \log q}\left[\langle u, 1\rangle \log |\cdot|_{K}-\left\langle u, \log |\cdot|{ }_{K}\right\rangle 1\right]
$$


hence $J$ is a rank 2 operator. We see from (3.11) that $\operatorname{tr} J=0$.

4) The only solution in $\mathcal{H}$ (up to the multiplication by a constant) of the equation $I_{0}^{1} u=0$ is the eigenfunction $u_{0}$ given by (3.8). Suppose that $\left(I_{0}^{1}\right)^{*} u_{0}=0$. Then $J u_{0}=0$. However by (3.12),

$$
\left(J u_{0}\right)\left(|x|_{K}\right)=\frac{1-q}{2 i q \log q} \log |x|_{K} \int_{|y|_{K}=1} d y=-\frac{(q-1)^{2}}{2 i q^{2} \log q} \log |x|_{K},
$$

so that $J u_{0} \not \equiv 0$, and we have come to a contradiction. This proves that $I_{0}^{1}$ is a simple Volterra operator.

Let us calculate the action of $I_{0}^{1}$ upon the basis $\left\{f_{n}\right\}$ defined in (2.10). We find for $|x|_{K}=$ $q^{-j}, j \geq 0$, that

$$
\begin{aligned}
\left(I_{0}^{1} f_{n}\right) & \left(|x|_{K}\right)=-\frac{\left(1-q^{-1}\right)^{1 / 2}}{\log q} q^{n / 2} \int_{|y|_{K}<q^{-j},|y|_{K}=q^{-n}}\left(\log |x|_{K}-\log |y|_{K}\right) d y \\
=\left(1-q^{-1}\right)^{1 / 2} q^{n / 2}(j-n) & \int_{|y|_{K}<q^{-j},|y|_{K}=q^{-n}} d y= \begin{cases}\left(1-q^{-1}\right)^{3 / 2} q^{-n / 2}(j-n), & \text { if } n>j ; \\
0, & \text { if } n \leq j .\end{cases}
\end{aligned}
$$

This implies the equality

$$
\left\langle I_{0}^{1} f_{n}, f_{j}\right\rangle=0 \text { for } n \leq j,
$$

meaning that $\left\{f_{n}\right\}$ is a basis of triangular representation for the operator $I_{0}^{1}$.

Remark. The operator $I_{0}^{1}$ is $S$-real with respect to the involution $S$ in $\mathcal{H}$ given by the complex conjugation. Therefore it is $S$-unicellular ([5], Appendix, Theorem 5.5). It is not clear whether it is unicellular in the usual (complex) sense. However it is unicellular in a smaller space $\mathcal{H}^{p}$ defined as a completion of the set of all "polynomials" $\varphi\left(|x|_{K}\right)=\sum_{j=0}^{N} c_{j}|x|_{K}^{j}$ with respect to the norm $\|\varphi\|=\left\{\sum\left|c_{j}\right|^{p}\right\}^{1 / p}, 1 \leq p<\infty$. By virtue of $\left([3.6), I_{0}^{1}\right.$ acts on the space $\mathcal{H}^{p}$ (isomorphic to $l^{p}$ ) as a weighted shift, for which the unicellularity was proved by Yakubovich [20].

3.4. Characteristic function. Following the notation in [6], let us write (3.13) in the form

$$
\frac{1}{i}\left(I_{0}^{1}-\left(I_{0}^{1}\right)^{*}\right) u=\sum_{\alpha, \beta=1}^{2}\left\langle u, h_{\alpha}\right\rangle j_{\alpha \beta} h_{\beta}
$$

where $h_{1}\left(|x|_{K}\right)=\frac{q-1}{i q \log q}(=$ const $), h_{2}\left(|x|_{K}\right)=-\log |x|_{K}, x \in O, j=\left(\begin{array}{ll}0 & 1 \\ 1 & 0\end{array}\right)$. For the operator $I_{0}^{1}$, we consider the $2 \times 2$ characteristic matrix-function of inverse argument

$$
W\left(z^{-1}\right)=E+i z j\left[\left\langle\left(E-z I_{0}^{1}\right)^{-1} h_{\alpha}, h_{\beta}\right\rangle\right]_{\alpha, \beta=1}^{2}
$$

where $E$ denotes both the unit operator in $\mathcal{H}$ and the unit matrix.

For the Volterra operator $I_{0}^{1}, W\left(z^{-1}\right)$ is an entire matrix-function. 
Theorem 3. Matrix elements of $W\left(z^{-1}\right)$ are entire functions of zero order.

Proof. For small values of $|z|$, the Fredholm resolvent $\left(E-z I_{0}^{1}\right)^{-1}$ is given by the Neumann series

$$
\left(E-z I_{0}^{1}\right)^{-1} f=\sum_{n=0}^{\infty}\left(z I_{0}^{1}\right)^{n} f, \quad f \in \mathcal{H} .
$$

In order to calculate the characteristic function, we have to compute the functions $\left(I_{0}^{1}\right)^{n} 1$ and $\left(I_{0}^{1}\right)^{n} \log |\cdot|_{K}$. The first of them is obtained easily from (3.6):

$$
\left(\left(I_{0}^{1}\right)^{n} 1\right)\left(|x|_{K}\right)=c^{n} \prod_{m=0}^{n-1} d_{m} \cdot|x|_{K}^{n}, \quad|x|_{K} \leq 1,
$$

where $c=\frac{1-q}{q \log q}, 0<d_{m} \leq A q^{-m}$. Summing the progression we find that

$$
\left(\left(E-z I_{0}^{1}\right)^{-1} 1\right)\left(|x|_{K}\right)=\sum_{n=0}^{\infty} \rho_{n} z^{n}|x|_{K}^{n}, \quad\left|\rho_{n}\right| \leq C^{n} q^{-n^{2} / 2},
$$

where $C>0$ is a constant.

Let us consider $\left(I_{0}^{1}\right)^{n} \log |\cdot|_{K}$. We have

$$
\left(I_{0}^{1} \log |\cdot|_{K}\right)\left(|x|_{K}\right)=c \int_{|y|_{K}<|x|_{K}}\left(\log |x|_{K}-\log |y|_{K}\right) \log |y|_{K} d y .
$$

Setting $y=x t,|t|_{K}<1$, we obtain

$$
\begin{aligned}
\left(I_{0}^{1} \log |\cdot|_{K}\right)\left(|x|_{K}\right)=-c|x|_{K} \int_{|t|_{K}<1} \log |t|_{K}\left(\log |x|_{K}+\log |t|_{K}\right) d t & =-c a_{0}|x|_{K} \log |x|_{k}-c b_{0}|x|_{K} \\
& \stackrel{\text { def }}{=} \sigma_{1}|x|_{K} \log |x|_{K}-\eta_{1}|x|_{K}
\end{aligned}
$$

where

$$
a_{0}=\int_{|t|_{K}<1} \log |t|_{K} d t, \quad b_{0}=\int_{|t|_{K}<1} \log ^{2}|t|_{K} d t .
$$

A similar calculation yields the expression

$$
\left(I_{0}^{1}\left(|\cdot|_{K} \log |\cdot|_{K}\right)\right)\left(|x|_{K}\right)=-c a_{1}|x|_{K}^{2} \log |x|_{k}-c b_{1}|x|_{K}^{2}
$$

where

$$
a_{1}=\int_{|t|_{K}<1}|t|_{K} \log |t|_{K} d t, \quad b_{1}=\int_{|t|_{K}<1}|t|_{K} \log ^{2}|t|_{K} d t .
$$

Together with (3.6), this implies the formula

$\left(\left(I_{0}^{1}\right)^{2} \log |\cdot|_{K}\right)\left(|x|_{K}\right)=c^{2} a_{0} a_{1}|x|_{K}^{2} \log |x|_{k}+c^{2} a_{0} b_{1}|x|_{K}^{2}-c b_{0} d_{1}|x|_{K}^{2} \stackrel{\text { def }}{=} \sigma_{2}|x|_{K}^{2} \log |x|_{K}+\eta_{2}|x|_{K}^{2}$. 
Introducing similar constants for the next iterations,

$$
a_{n}=\int_{|t|_{K}<1}|t|_{K}^{n} \log |t|_{K} d t, \quad b_{n}=\int_{|t|_{K}<1}|t|_{K}^{n} \log ^{2}|t|_{K} d t
$$

and noticing that $\left|a_{n}\right|,\left|b_{n}\right| \leq M q^{-n}$, we prove by induction that

$$
\left(I_{0}^{1}\right)^{n} \log |\cdot|_{K}=\sigma_{n}|x|_{K}^{n} \log |x|_{K}+\eta_{n}|x|_{K}^{n}
$$

where $\left|\sigma_{n}\right|,|\eta|_{n} \leq C^{n} q^{-1} q^{-2} \cdots q^{-n+1}=C^{n} q^{-n(n-1) / 2}$.

It follows from (3.15) that

$$
\left(\left(E-z I_{0}^{1}\right)^{-1} \log |\cdot|_{K}\right)\left(|x|_{K}\right)=\sum_{n=0}^{\infty} \sigma_{n} z^{n}|x|_{K}^{n} \log |x|_{K}+\sum_{n=0}^{\infty} \eta_{n} z^{n}|x|_{K}^{n}
$$

where $\left|\sigma_{n}\right|,\left|\eta_{n}\right| \leq C_{1}^{n} q^{-n^{2} / 2}$.

Now we can compute the matrix-function $W\left(z^{-1}\right)$. By (3.14),

$$
\left\langle\left(E-z I_{0}^{1}\right)^{-1} h_{1}, h_{1}\right\rangle=\mathrm{const} \cdot \sum_{n=0}^{\infty} \rho_{n} z^{n} \int_{|x|_{K}^{n} \leq 1} d x=\sum_{n=0}^{\infty} \gamma_{n} z^{n}
$$

where $\left|\gamma_{n}\right| \leq C_{2}^{n} q^{-n^{2} / 2}$, so that this matrix element is an entire function of zero order. Other matrix elements are estimated similarly on the basis of (3.15), by inserting 1 as an upper bound of $|x|_{K}$ and taking into account the convergence of the integrals of log $|x|_{K}$ and $\log ^{2}|x|_{K}$.

\section{The Laplace Type Transform}

4.1. Definition and Properties. Our definition of a Laplace type transform is based on the function $v_{N}$ given by (2.6). It is essential that $v_{N} \in \mathcal{D}(K)$. As we know, $D^{\alpha} v_{N}=q^{\alpha N} v_{N}$ $(\alpha>0)$.

Let $\xi \in K,|\xi|_{K}=q^{N}$. Then for any $x \in K, v_{N}\left(|x|_{K}\right)=v_{0}\left(|x \xi|_{K}\right)$,

$$
D_{x}^{\alpha} v_{0}\left(|x \xi|_{K}\right)=D_{x}^{\alpha} v_{N}\left(|x|_{K}\right)=q^{\alpha N} v_{N}\left(|x|_{K}\right)=|\xi|_{K}^{\alpha} v_{0}\left(|x \xi|_{K}\right) .
$$

We call the function

$$
\widetilde{\varphi}\left(|\xi|_{K}\right)=\int_{K} v_{0}\left(|x \xi|_{K}\right) \varphi\left(|x|_{K}\right) d x
$$

the Laplace type transform of a radial function $\varphi \in L_{\text {loc }}^{1}(K)$. By the dominated convergence theorem, $\widetilde{\varphi}$ is continuous, bounded, and $\widetilde{\varphi}\left(|\xi|_{K}\right) \rightarrow 0,|\xi|_{K} \rightarrow \infty$.

As a simple computation shows, if $\varphi\left(|x|_{K}\right) \equiv$ const, then $\widetilde{\varphi}\left(|\xi|_{K}\right) \equiv 0$.

The above calculations, together with the selfadjointness of $D^{\alpha}$ in $L^{2}(K)$, show that

$$
\widetilde{D^{\alpha} \varphi}\left(|\xi|_{K}\right)=\int_{K}\left(D_{x}^{\alpha} v_{0}\left(|x \xi|_{K}\right)\right)\left(|x|_{K}\right) \varphi\left(|x|_{K}\right) d x=|\xi|_{K}^{\alpha} \widetilde{\varphi}\left(|\xi|_{K}\right), \quad \xi \in K .
$$


Theorem 4 (uniqueness). If $\widetilde{\varphi}\left(|\xi|_{K}\right) \equiv 0$, then $\varphi\left(|x|_{K}\right) \equiv$ const.

Proof. By the definition,

$$
\widetilde{\varphi}\left(|\xi|_{K}\right)=\int_{|x|_{K} \leq|\xi|_{K}^{-1}} \varphi\left(|x|_{K}\right) d x-\frac{1}{q-1} \int_{|x|_{K}=q|\xi|_{K}^{-1}} \varphi\left(|x|_{K}\right) d x .
$$

Let $|\xi|_{K}=q^{n}, n \in \mathbb{Z}$. Then

$$
\widetilde{\varphi}\left(q^{n}\right)=\left(1-\frac{1}{q}\right) \sum_{j=-\infty}^{-n} \varphi\left(q^{j}\right) q^{j}-\varphi\left(q^{-n+1}\right) q^{-n}
$$

If we denote $\widetilde{\varphi}\left(q^{n}\right)=f_{n}$, then

$$
f_{n+1}=\left(1-\frac{1}{q}\right) \sum_{j=-\infty}^{-n-1} \varphi\left(q^{j}\right) q^{j}-\varphi\left(q^{-n}\right) q^{-n-1}
$$

so that

$$
f_{n}-f_{n+1}=q^{-n}\left[\varphi\left(q^{-n}\right)-\varphi\left(q^{-n+1}\right)\right] .
$$

If $\widetilde{\varphi}\left(q^{n}\right)=0$ for all $n$, then, by (4.1), $\varphi\left(q^{-n}\right)=\varphi\left(q^{-n+1}\right)$ for all $n$, so that $\varphi\left(q^{-n}\right) \equiv$ const.

The identity (4.1) is of some independent interest, and we formulate it as a corollary.

Corollary 1. For all $n \in \mathbb{Z}$,

$$
\widetilde{\varphi}\left(q^{n}\right)-\widetilde{\varphi}\left(q^{n+1}\right)=q^{-n}\left[\varphi\left(q^{-n}\right)-\varphi\left(q^{-n+1}\right)\right] .
$$

Corollary 2. A function $\varphi$ is (strictly) monotone, if and only if $\widetilde{\varphi}$ is (strictly) monotone.

\subsection{Inversion formula.}

Theorem 5. For each $n=1,2, \ldots$,

$$
\begin{gathered}
\varphi\left(q^{m}\right)=\varphi(1)+\sum_{j=0}^{m-1} q^{-j}\left[\widetilde{\varphi}\left(q^{-j+1}\right)-\widetilde{\varphi}\left(q^{-j}\right)\right] \\
\varphi\left(q^{-m}\right)=\varphi(1)+\sum_{j=1}^{m} q^{j}\left[\widetilde{\varphi}\left(q^{j}\right)-\widetilde{\varphi}\left(q^{j+1}\right)\right]
\end{gathered}
$$


Proof. According to (4.2),

$$
\begin{gathered}
\varphi(1)-\varphi(q)=\widetilde{\varphi}(1)-\widetilde{\varphi}(q), \\
\varphi(q)-\varphi\left(q^{2}\right)=q^{-1}\left[\widetilde{\varphi}\left(q^{-1}\right)-\widetilde{\varphi}(1)\right], \\
\varphi\left(q^{2}\right)-\varphi\left(q^{3}\right)=q^{-2}\left[\widetilde{\varphi}\left(q^{-2}\right)-\widetilde{\varphi}\left(q^{-1}\right)\right],
\end{gathered}
$$

etc. Summing up the first $m$ equalities we obtain (4.3).

Similarly, by (4.2),

$$
\begin{aligned}
\varphi\left(q^{-1}\right)-\varphi(1) & =q\left[\widetilde{\varphi}(q)-\widetilde{\varphi}\left(q^{2}\right)\right], \\
\varphi\left(q^{-2}\right)-\varphi\left(q^{-1}\right) & =q^{2}\left[\widetilde{\varphi}\left(q^{2}\right)-\widetilde{\varphi}\left(q^{3}\right)\right],
\end{aligned}
$$

etc, and the summation yields (4.4).

\section{Acknowledgement}

This work was funded in part under the research project "Markov evolutions in real and $p$-adic spaces" of the Dragomanov National Pedagogic University of Ukraine.

\section{References}

[1] S. Albeverio, A. Yu. Khrennikov, and V. M. Shelkovich, Theory of p-Adic Distributions, Cambridge University Press, 2010.

[2] A. D. Bendikov, A. A. Grigor'yan, Ch. Pittet, W. Woess, Isotropic Markov semigroups on ultra-metric spaces, Russian Math. Surveys, 69 (2014), 589-680.

[3] Bo Wu and A. Yu. Khrennikov, p-Adic analogue of the wave equation, J. Fourier Anal. Appl. 25 (2019), 2447-2462.

[4] M. S. Brodskii, Triangular and Jordan representations of Linear Operators. American Mathematical Society, Providence, R.I., 1971.

[5] I. C. Gohberg and M. G. Krein, Theory and Applications of Volterra Operators in Hilbert Space. American Mathematical Society, Providence, R.I. 1970.

[6] G. M. Gubreev and A. A. Tarasenko, Spectral decomposition of model operators in de Branges spaces, Sbornik Math. 201 (2010), 1599-1634.

[7] P. R. Halmos, Hilbert Space Problem Book, D. Van Nostrand, Princeton, 1967.

[8] A. Yu. Khrennikov, S. V. Kozyrev and W. A. Zúñiga-Galindo, Ultrametric Pseudodifferential Equations with Applications, Cambridge University Press, 2018.

[9] A. Yu. Khrennikov and A. N. Kochubei, p-Adic analogue of the porous medium equation. J. Fourier Anal. Appl. 24 (2018), 1401-1424. 
[10] A. Yu. Khrennikov and A. N. Kochubei, On the p-adic Navier-Stokes equation. Applicable Anal. 99 (2020), no. 8, 1425-1435.

[11] A. N. Kochubei, Pseudo-Differential Equations and Stochastics over Non-Archimedean Fields, Marcel Dekker, New York, 2001.

[12] A. N. Kochubei, A non-Archimedean wave equation, Pacif. J. Math. 235 (2008), 245-261.

[13] A. N. Kochubei, Radial solutions of non-Archimedean pseudodifferential equations, Pacif. J. Math. 269 (2014), 355-369.

[14] A. N. Kochubei, Linear and nonlinear heat equations on a p-adic ball, Ukrainian Math. J. 70 (2018), 217-231.

[15] A. N. Kochubei, Nonlinear pseudo-differential equations for radial real functions on a non-Archimedean field. J. Math. Anal. Appl. 483 (2020), no. 1, Article 123609, 11 pp.

[16] M. M. Malamud, Spectral theory of fractional order integration operators, their direct sums, and similarity problem to these operators of their weak perturbations. In: Handbook of fractional calculus with applications. Vol. 1 (A. N. Kochubei and Yu. Luchko, Eds), De Gruyter, Berlin, 2019, pp. 427-460.

[17] K. Oleschko, A. Khrennikov and M. J. Correa López, p-Adic analog of Navier-Stokes equations: Dynamics of fluid's flow in percolation networks, Entropy 19 (2017), Paper No. 161, 14 pp.

[18] E. Pourhadi, A. Khrennikov et al, Solvability of the p-adic analogue of Navier-Stokes equation via the wavelet theory. Entropy 21 (2019), no. 11, Paper No. 1129, 20 pp.

[19] V. S. Vladimirov, I. V. Volovich and E. I. Zelenov, p-Adic Analysis and Mathematical Physics, World Scientific, Singapore, 1994.

[20] D. V. Yakubovich, Invariant subspaces of weighted shift operators, J. Soviet Math. 37 (1987), 1323-1346.

[21] V. A. Zolotarev, L. de Branges spaces and functional models of nondissipative operators, Mat. Fiz. Anal. Geom. 9 (2002), 622-641 (Russian).

[22] W. A. Zúñiga-Galindo, Pseudodifferential Equations over Non-Archimedean Spaces, Lect. Notes Math. 2174, Springer, Cham, 2016. 